\title{
Hypoxia treatment on germinating faba bean (Vicia faba L.) seeds enhances GABA-related protection against salt stress
}

\author{
Runqiang Yang ${ }^{1}$, Shufang Wang ${ }^{1}$, Yongqi Yin ${ }^{1}$, and Zhenxin Gu${ }^{1 *}$
}

\begin{abstract}
The $\gamma$-aminobutyric acid (GABA) is a non-protein amino acid with some functional properties for human health. Its content is usually lower in plant seeds. Hypoxia or salt $(\mathrm{NaCl})$ stress is an effective way for accumulating GABA during seed germination. However, $\mathrm{NaCl}$ stress on $\mathrm{GABA}$ accumulation under hypoxia is currently infrequent. The effect of $\mathrm{NaCl}$ on GABA accumulation in germinating faba bean (Vicia faba L.) under hypoxia was therefore investigated in this study. Faba bean seeds were steeped in citric acid buffer $(\mathrm{pH} 3.5)$ containing $\mathrm{NaCl}$ with a final $\mathrm{O}_{2}$ concentration of 5.5 mg $\mathrm{L}^{-1}$ and germinated for $5 \mathrm{~d}$. Results showed that $60 \mathrm{mmol} \mathrm{L}^{-1} \mathrm{NaCl}$ was the optimum concentration for GABA accumulation in germinating faba beans under hypoxia. Germination for $5 \mathrm{~d}$ under hypoxia- $\mathrm{NaCl}$ stress was less beneficial for $\mathrm{GABA}$ accumulation than only hypoxia (control). Polyamine degradation pathway played a more important role for accumulating GABA in germinating faba bean as an adaptive response to $\mathrm{NaCl}$ stress. Removing $\mathrm{NaCl}$ significantly increased $\mathrm{GABA}$ content, while it decreased glutamate decarboxylase (GAD) activity. Simultaneously, polyamine was accumulated, which might be related to the enhancement of physiological activity after recovery. When treated with aminoguanidine (AG) for 3 d, GABA content decreased by $29.82 \%$. These results indicated that the tolerance ability of GABA shunt to $\mathrm{NaCl}$ stress was weaker than that of polyamine degradation pathway. The $\mathrm{NaCl}$ treatment for $3 \mathrm{~d}$ under hypoxia could raise the contribution ratio of polyamine degradation pathway for GABA accumulation. The contribution ratio of polyamine degradation pathway for GABA formation was $29.82 \%$ when treated for at least $3 \mathrm{~d}$.
\end{abstract}

Key words: GABA shunt, germination, polyamine degradation pathway, Vicia faba.

\section{INTRODUCTION}

The $\gamma$-aminobutyric acid (GABA) is a non-protein amino acid acting as a neurotransmitter in the brain of mammals (Kinnersley and Turano, 2000) and exists widely in nature. Plant GABA content is usually low, while it increases significantly when the plant is subjected to abiotic stress. In recent years, the development of GABA-enriched products has attracted significant interest because of their functional effects on human health (Mody et al., 1994). In plants, GABA can be synthesized by two pathways. One is called GABA shunt where $\alpha$-decarboxylation of L-glutamate (L-Glu) is catalyzed by glutamate decarboxylase (GAD, EC 4.1.1.15) (Bown and Shelp, 1997); the other is polyamine degradation pathway where diamine oxidase (DAO, EC 1.4.3.6) is the key enzyme (Bhatnagar et al., 2001). Current research on GABA accumulation in plants has mainly been focused on GABA shunt (Bai et al., 2009; Li et al., 2010), and limited to polyamine degradation pathway (Yang et al., 2013).

${ }^{1}$ Nanjing Agricultural University, College of Food Science and Technology, Nanjing, Jiangsu, 210095, People's Republic of China.

"Corresponding author (guzx@njau.edu.cn).

Received: 12 August 2014.

Accepted: 12 December 2014.

doi:10.4067/S0718-58392015000200007
Faba bean (Vicia faba L.) is a common legume that is rich in nutritive substances. However, its use is largely limited by the presence of anti-nutritional compounds, such as phytic acid (Oomah et al., 2011), tannins, vicine, convicine (Goyoaga et al., 2008; Vilariño et al., 2009), and trypsin inhibitors (Guillamon et al., 2008). Germination could improve the nutritional components and decrease the level of anti-nutritional factors in legumes (Azeke et al., 2011). New functional components, such as GABA, are generated (Li et al., 2010) during seed germination. Previous research studies reported that stressful conditions (salt: Xing et al., 2007; hypoxia, Guo et al., 2011; 2012; heat: Wellner et al., 2011; water stress: Misra et al., 2002; and cold shock: Bouche et al., 2003) could strongly enhance GAD and DAO activities and accumulate GABA in plants. However, these research studies only discussed one type of stressful condition. It is unknown whether adding another type of stress combined with the existing stress could result in an additional increase in GABA content. Studies about the relationship between DAO activity and GABA accumulation have been sparsely reported (Xing et al., 2007). Aminoguanidine (AG) can block polyamine degradation pathway by inhibiting DAO activity. This provides an effective way for studying the contribution ratio of two pathways for GABA accumulation. 
In this study, we investigated the effect of $\mathrm{NaCl}$ on GABA accumulation in germinating faba bean under hypoxia. The objectives were to clarify the role of GABA shunt and polyamine degradation pathway on GABA accumulation under $\mathrm{NaCl}$ stress and investigate the combined stress of hypoxia and $\mathrm{NaCl}$ for GABA accumulation in germinating faba bean.

\section{MATERIALS AND METHODS}

Faba bean seeds ('Qi Bean 2'), harvested in 2012, were purchased from Jiangsu Academy of Agricultural Sciences (Nanjing, China) and stored at $-20{ }^{\circ} \mathrm{C}$ prior to the experiment. Horseradish peroxidase, putrescine (Put), spermidine (Spd), spermine (Spm), 4-aminoantipyrine, $\mathrm{N}, \mathrm{N}$-dimethylaniline, dimethylaminoazobenzene sulfonyl chloride (dabsyl chloride, 99\%), and aminoguanidine (AG) were purchased from Sigma Chemical (St Louis, Missouri, USA). All other reagents were analytical grade.

\section{Pretreatment of seeds}

Dried seeds were sterilized with $10 \mathrm{~mL} \mathrm{~L}^{-1}$ sodium hypochlorite for $30 \mathrm{~min}$ and then washed and steeped in deionized water at $30{ }^{\circ} \mathrm{C}$ for $8 \mathrm{~h}$. Soaked seeds were put into the culturing pallet $(25 \mathrm{~cm}$ length $\times 20 \mathrm{~cm}$ width $\times 5$ $\mathrm{cm}$ height) and cultured for $48 \mathrm{~h}$ in a dark incubator (30 $\left.{ }^{\circ} \mathrm{C}\right)$. Deionized water was provided every $8 \mathrm{~h}$ to complete the start of seed metabolism.

To select the $\mathrm{NaCl}$ concentration after pretreatment, seeds were drained and placed in cultivated pots with lids $(\phi 6.0 \mathrm{~cm} \times 9.0 \mathrm{~cm})$ where they were germinated with citrate buffer $\left(10 \mathrm{mmol} \mathrm{L}^{-1}, \mathrm{pH} 3.5\right)$ containing 0 , $20,40,60,80$, and $100 \mathrm{mmol} \mathrm{L}^{-1} \mathrm{NaCl}$ at $30{ }^{\circ} \mathrm{C}$ for $3 \mathrm{~d}$. The culture solution was aerated with a pump (Yuyao Jintai Meter Co. Ltd., Zhejiang, China). The air flow rate $\left(1.2 \mathrm{~L} \mathrm{~min}^{-1}\right)$ was controlled with a flow meter $(5.5$ $\mathrm{mg} \mathrm{L}^{-1}$ final dissolved oxygen concentration). The culture solution was replaced with a fresh solution every $12 \mathrm{~h}$ until germination was completed. The GABA content of the sprout was then analyzed.

\section{Treatments and recovery from stress}

To impose hypoxia stress (control) after pretreatment, seeds were treated for 0,3 , and $5 \mathrm{~d}$ as already described for $\mathrm{NaCl}$ concentration selection, but without adding $\mathrm{NaCl}$. The final dissolved oxygen concentration was 5.5 $\mathrm{mg} \mathrm{L}^{-1}$. The culture solution was replaced with a fresh solution every $12 \mathrm{~h}$ until germination was completed.

To study the effect of $\mathrm{NaCl}$ stress after pretreatment, seeds were germinated with the same method as described for hypoxia stress (control), with the exception that the culture solution contained $60 \mathrm{mmol} \mathrm{L}^{-1} \mathrm{NaCl}$.

To evaluate stress recovery, faba bean seeds were treated for $2 \mathrm{~d}$ as already described for $\mathrm{NaCl}$ stress. They were then cultured for $3 \mathrm{~d}$ as described for hypoxia stress (control).
The germination procedure was the same as the one previously described for $\mathrm{NaCl}$ stress, with the exception that the culture solution contained $7.5 \mathrm{mmol}$ aminoguanidine (AG) $\mathrm{L}^{-1}$. Germinated seeds were rinsed with distilled water, dried on filter paper, frozen in liquid nitrogen, and then analyzed for GAD and DAO activity, and GABA, Glu, and polyamine content.

\section{Chemical analyses}

The GABA and Glu contents were analyzed according to Yang et al. (2013). Germinated faba bean (1.00 g) was mulled with $6 \mathrm{~mL}$ of $4 \%(\mathrm{v} / \mathrm{v})$ acetic acid. The homogenate was deposited for $1 \mathrm{~h}$ to extract sufficient GABA and then centrifuged at $6037 \mathrm{~g}$ for $15 \mathrm{~min}$. The supernatant was collected and treated with $4 \mathrm{~mL}$ ethanol to remove macro-molecular polymers and then centrifuged at 11200 $\mathrm{g}$ for $20 \mathrm{~min}$. The purified supernatant was evaporated $\left(0.1 \mathrm{MPa}, 45^{\circ} \mathrm{C}\right)$ to volatilize the acetic acid and ethanol. Residues were dissolved with $2 \mathrm{~mL}$ of $1 \mathrm{~mol} \mathrm{~L}^{-1} \mathrm{NaHCO}_{3}$ (pH 9.0) and centrifuged at $6000 \mathrm{~g}$ for $10 \mathrm{~min}$. The amino acid solution ( $1 \mathrm{~mL}, \mathrm{pH} 9.0)$ was mixed with $1 \mathrm{~mL}$ dabsyl chloride $\left(2 \mathrm{mg} \mathrm{mL}^{-1}\right.$, in acetone $)$ and reacted at $67^{\circ} \mathrm{C}$ for $10 \mathrm{~min}$. The reaction was stopped by putting the tubes in an ice bath. Both GABA and Glu were determined by high performance liquid chromatography (HPLC; Agilent 1200 LC, Santa Clara, California, USA) with a ZORBAX Eclipse AAA reversed-phase column $(3.5 \mu \mathrm{m}), 4.6 \times 150$ $\mathrm{mm}$ id, at $425 \mathrm{~nm}$ by UV-vis diode-array absorbance detection (DAD). Mobile phase A was acetonitrile and mobile phase $\mathrm{B}$ was $0.045 \mathrm{~mol} \mathrm{~L}^{-1} \mathrm{CH}_{3} \mathrm{COONa}(\mathrm{pH} 4)$; the allowed separation time of GABA and Glu was within $30 \mathrm{~min}$ at a constant temperature of $30^{\circ} \mathrm{C}$.

Free polyamines were analyzed by HPLC as described by Xing et al. (2007). Benzoyl-polyamines ( $20 \mu \mathrm{L})$ were analyzed by HPLC (Agilent 1200) with a ZORBAX Eclipse AAA reversed-phase column $(3.5 \mu \mathrm{m}) ; 4.6 \times$ $150 \mathrm{~mm}$ id, and methanol/ $\mathrm{H}_{2} \mathrm{O}(64: 36)(\mathrm{v} / \mathrm{v})$ was used as an isocratic eluting solvent at a $0.6 \mathrm{~mL} \mathrm{~min}^{-1}$ flow rate. Polyamine content was expressed as nmol g ${ }^{-1}$ or $\mu \mathrm{mol} \mathrm{g} \mathrm{g}^{-1}$ dry weight (DW). The HPLC profile of free polyamines in germinated faba bean is shown in Figure 1.

The GAD activity was determined according to Bai et al. (2009) and Guo et al. (2012). One unit of GAD activity was defined as the release of $1 \mu \mathrm{mol}$ GABA per $60 \mathrm{~min}$ at $40{ }^{\circ} \mathrm{C}$. The GAD activity of plant tissues is defined as 1 $\mathrm{mg}$ protein units of GAD activity. The DAO activity was determined according to Yang et al. (2011a). A 0.01 value of the changes per minute in absorbance at $555 \mathrm{~nm}$ was regarded as one unit of enzyme activity.

Protein content was measured from the supernatant using a protein-dye reagent (Coomassie blue G-250) with bovine serum albumin as a standard according to Yang et al. (2011b). 

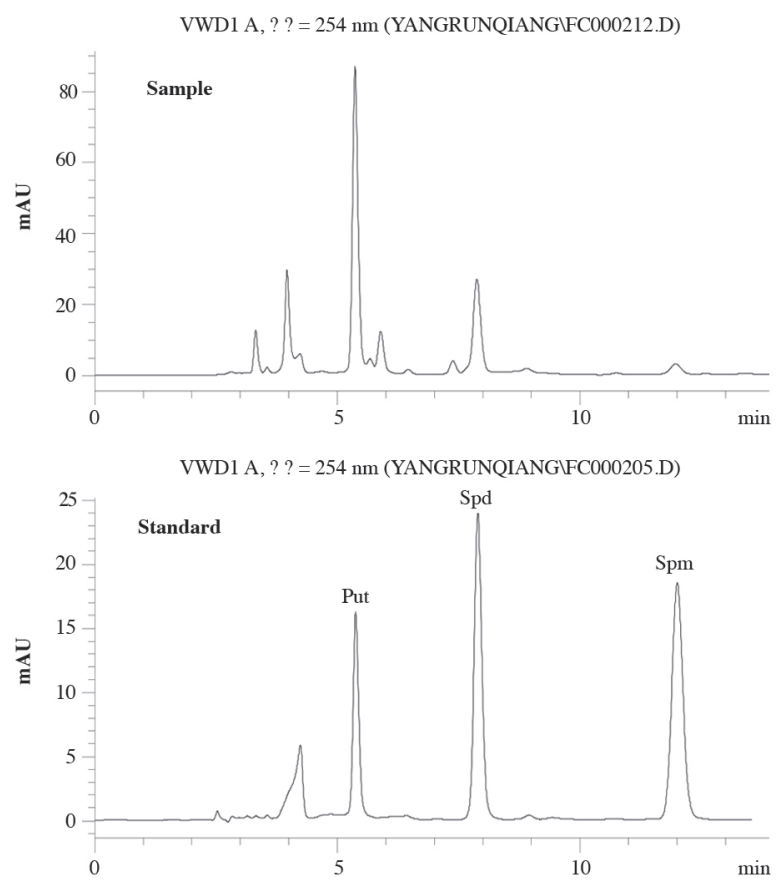

Figure 1. High performance liquid chromatography (HPLC) profile of polyamines-putrescine (Put), spermidine (Spd), and spermine $(\mathrm{Spm})$.

\section{Statistical analysis}

Mean values and standard deviations were computed in accordance with experimental data. Statistical analysis was performed by Fisher's $F$-test; $p<0.05$ was considered significant.

\section{RESULTS}

\section{$\mathrm{NaCl}$ stress}

At first, GABA content increased and then decreased with increasing $\mathrm{NaCl}$ concentrations, reaching peak value at 60 and $80 \mathrm{mmol} \mathrm{L}^{-1} \mathrm{NaCl}$ (Figure 2). The lower concentration was not able to increase GABA content,

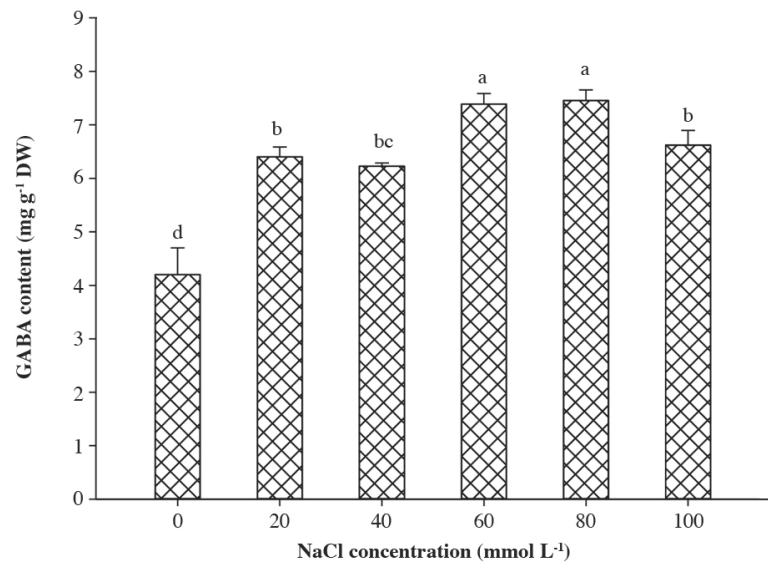

Figure 2. Effect of $\mathrm{NaCl}$ concentration on $\gamma$-aminobutyric acid (GABA) accumulation of germinating faba bean under hypoxia. Each point was expressed as the mean \pm SD. Different lowercase letters represent significant differences at $p<0.05$. while a higher concentration inhibited seed survival and thus slowed down the increase in GABA content. Hence, to conserve material, $60 \mathrm{mmol} \mathrm{L}^{-1} \mathrm{NaCl}$ was selected for GABA accumulation in germinating faba bean under hypoxia.

Germination produced a significant increase in GABA content of germinating faba beans (Figure $3 \mathrm{~A})$. Salt $(\mathrm{NaCl})$ stress increased GABA content by 1.05 - and 0.20 -fold in the cotyledon and embryo of 3-d-old sprouts, respectively (Figure 3B) compared with the control (under hypoxia). Via the $\alpha$-decarboxylation process, GAD converts L-Glu into GABA. Hence, GAD activity is vital for GABA accumulation. When germinated for $5 \mathrm{~d}$ under $\mathrm{NaCl}$ stress, GAD activity was inhibited significantly as compared with the control (Figure 3C). No change in GAD activity was observed in the cotyledon of 3-d-old sprouts, while it decreased significantly in the embryo (Figure 3D). For DAO activity, the variation trend was similar to that of GAD activity (Figure 3E), but it was higher than that of the control in the embryo (Figure $3 \mathrm{~F}$ ).

Glutamate is converted into GABA by GAD. Polyamines, especially putrescine (Put) and spermidine
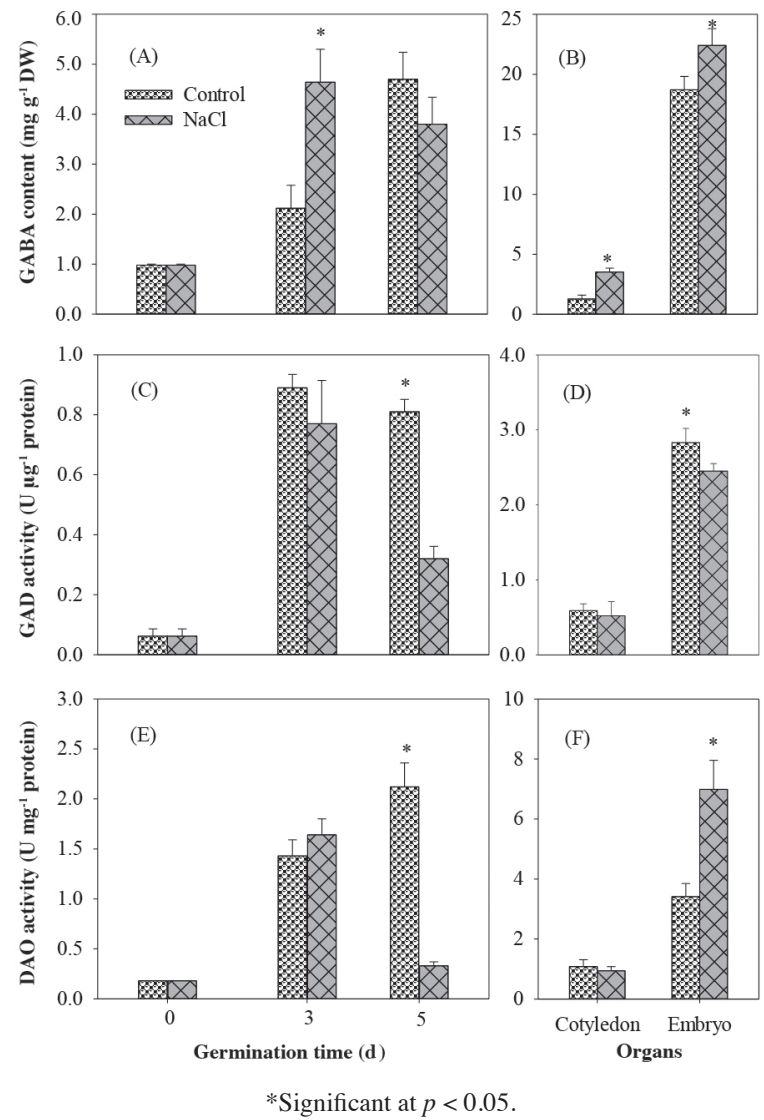

Figure 3. Effect of NaCl on $\gamma$-aminobutyric acid (GABA) content $(\mathrm{A}, \mathrm{B})$, glutamate decarboxylase (GAD; C, D), and diamine oxidase (DAO; E, F) activity of germinating faba bean under hypoxia. Seeds germinating under hypoxia were defined as the control. Indexes in the cotyledon and embryo of 3-d-old sprouts were determined. Each point was expressed as mean \pm SD. 
(Spd), are catalyzed by DAO via an intermediate ( $\gamma$-aminobutylaldehyde) that is converted into GABA. Figure 4A shows that there were no significant changes in Glu content compared with the control (under hypoxia) when germinated for $5 \mathrm{~d}$ under $\mathrm{NaCl}$ stress $(p>0.05)$. However, Glu content was less than the control; a similar trend occurred in the cotyledon and embryo of 3-d-old sprouts under $\mathrm{NaCl}$ stress (Figure 4B). Putrescine is the most important substrate of DAO; its content was higher in seeds germinated for $5 \mathrm{~d}$ and treated with $\mathrm{NaCl}$ as compared with the control (Figure 4C). When germination time was $3 \mathrm{~d}$, Put decreased slightly in the embryo under $\mathrm{NaCl}$ (Figure 4D). The changing trend of Spd content was similar to Put (Figure 4E); no significant changes existed in the cotyledon and embryo as compared with the control, respectively (Figure 4F). Spermine was accumulated when treated with $\mathrm{NaCl}$ for 3 and $5 \mathrm{~d}$ (Figure $4 \mathrm{G})$; it increased in the cotyledon, but decreased slightly in the embryo of 3-d-old sprouts (Figure 4H).

\section{Recovery from $\mathrm{NaCl}$ stress}

The present results showed that GABA accumulation was significant in faba bean when treated with $\mathrm{NaCl}$ under hypoxia. However, the changes in GABA content are unclear after eliminating the $\mathrm{NaCl}$ treatment. The recovery experiment was conducted to discuss the effect of $\mathrm{NaCl}$ on $\mathrm{GABA}$ accumulation in germinating faba bean under hypoxia stress.

Recovery after treatment with $\mathrm{NaCl}$ for $2 \mathrm{~d}$ and germination for $3 \mathrm{~d}$ under hypoxia stress increased GABA content significantly (Figure 5A); it mainly occurred in the embryo (Figure 5B). Recovery did not affect GAD
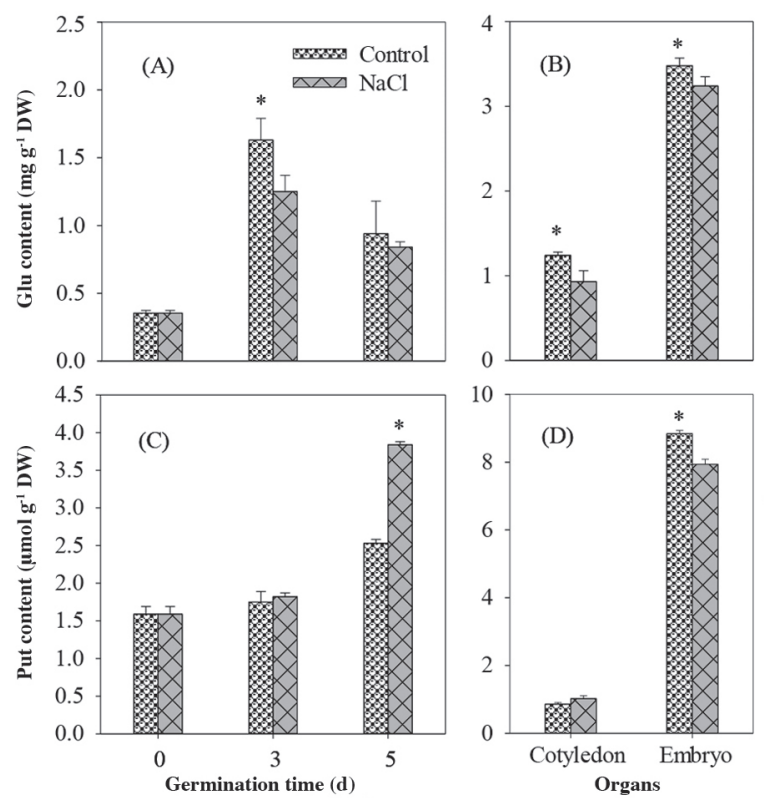

*Significant at $p<0.05$.
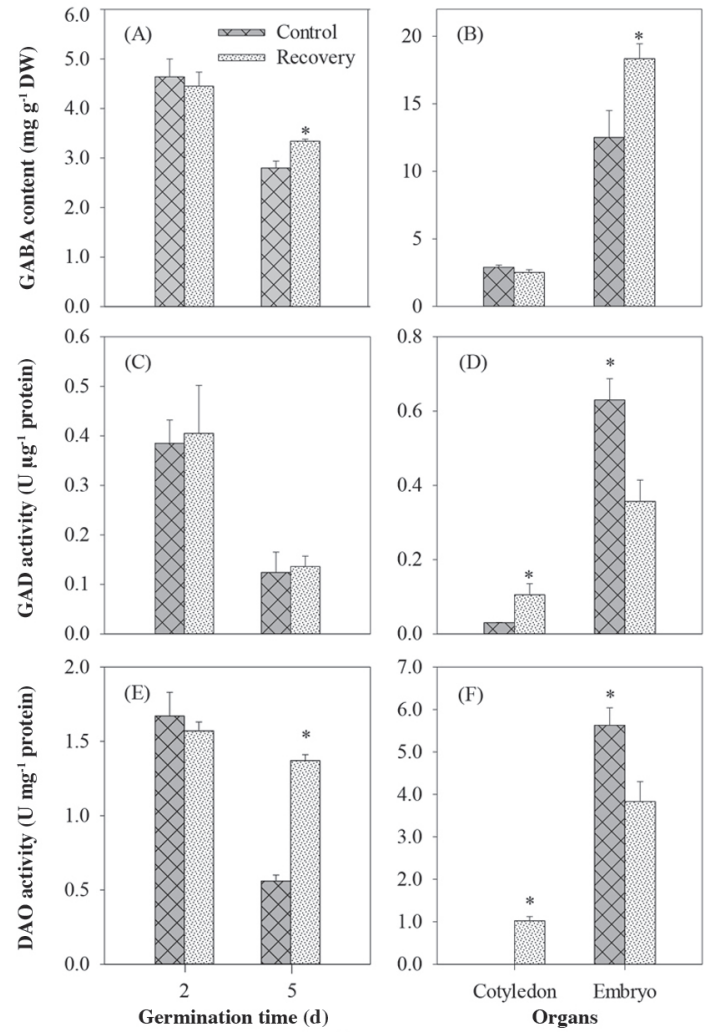

*Significant at $p<0.05$.

Figure 5. Effect of removing $\mathrm{NaCl}$ on $\gamma$-aminobutyric acid (GABA) content (A, B), glutamate decarboxylase (GAD; C, D), and diamine oxidase (DAO; E, F) activity of germinating faba bean under hypoxia. Seeds germinating under hypoxia were defined as the control. Indexes in the cotyledon and embryo were determined. Each point was expressed as mean $\pm \mathrm{SD}$.
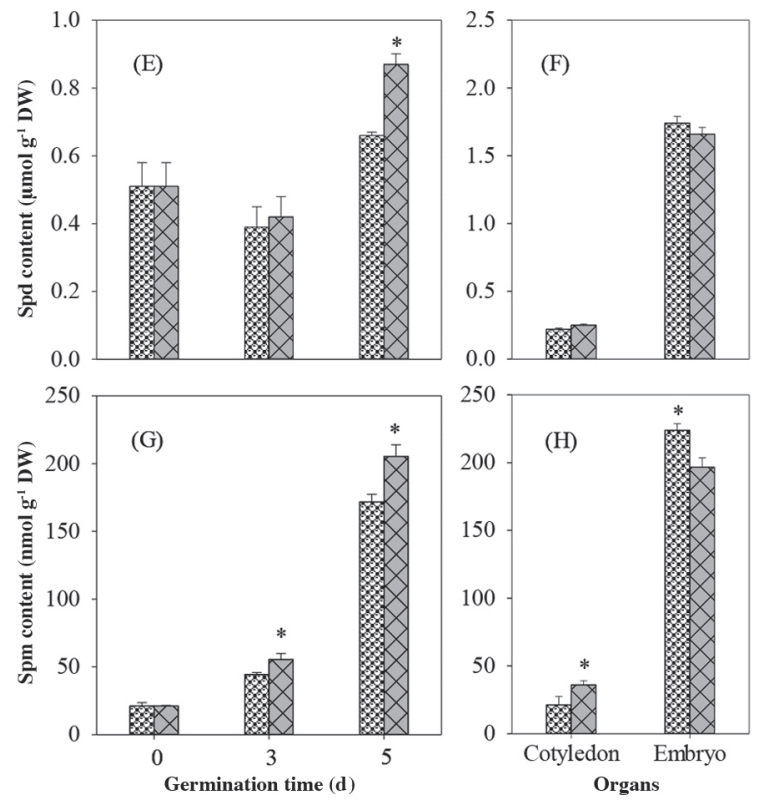

Figure 4. Effect of $\mathrm{NaCl}$ on glutamate (Glu; A, B) and polyamine (C, D, E, F, G, and H) content of germinating faba bean under hypoxia. Seeds germinating under hypoxia were defined as the control. Indexes in the cotyledon and embryo of 3-d-old sprouts were determined. Each point was expressed as mean $\pm \mathrm{SD}$. 
activity in sprouts (Figure 5C), but increased GAD activity in the cotyledon and decreased activity in the embryo (Figure 5D). After recovery, DAO activity increased by $59.12 \%$ (Figure 5E). The variation trend in the cotyledon and embryo was similar to GAD (Figure 5F).

The effect of recovery on Glu (Figure 6A, 6B) and Put (Figure 6C) content was not significant (Figure 6C). However, Put content decreased in the cotyledon, but increased in the embryo (Figure 6D). Spermidine content increased significantly (Figure 6E) in both the cotyledon and embryo (Figure 6F). Spermine content decreased significantly after recovery (Figure 6G), and occurred mainly in the cotyledon (Figure $6 \mathrm{H}$ ).

\section{Hypoxia-NaCl stress}

Aminoguanidine is a specific inhibitor of DAO; when added during seed germination, it can inhibit DAO activity. It is therefore necessary to investigate the contribution ratio of GABA shunt and polyamine degradation pathway on GABA accumulation.

Adding $\mathrm{AG}$ under hypoxia- $\mathrm{NaCl}$ stress decreased GABA content by $29.82 \%$ in faba bean treated for $3 \mathrm{~d}$ compared with the control (Figure 7A), and it mainly occurred in the embryo (Figure 7B). The GAD activity also significantly decreased and was mainly in the embryo (Figure 7C and 7D). The reason might be that adding AG inhibited the growth activity of faba bean (it was found that AG can inhibit radicle extension). The DAO activity was inhibited by $76.83 \%$ when AG was treated for $3 \mathrm{~d}$ under hypoxia- $\mathrm{NaCl}$ stress (Figure 7E); it was inhibited by $30.44 \%$ in the embryo and completely inhibited in
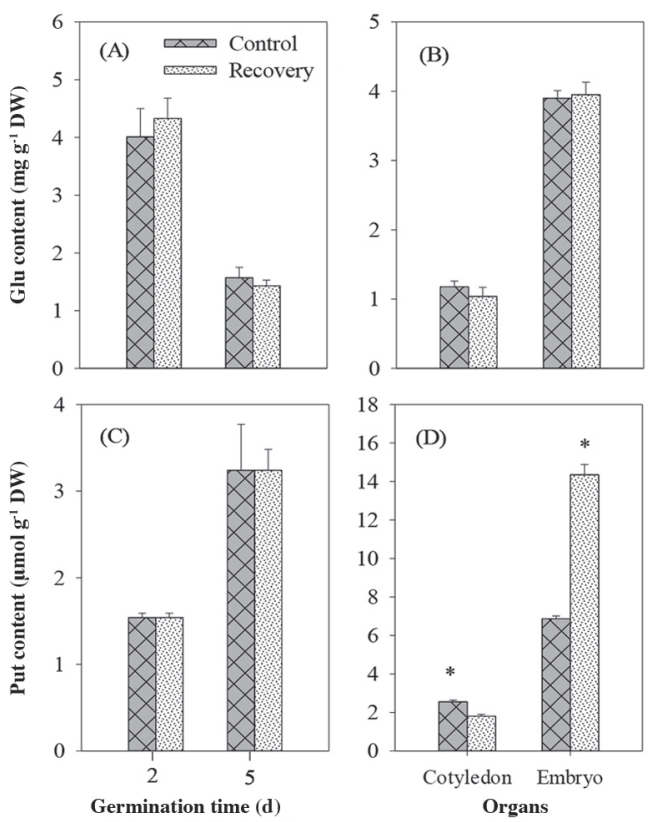

*Significant at $p<0.05$.
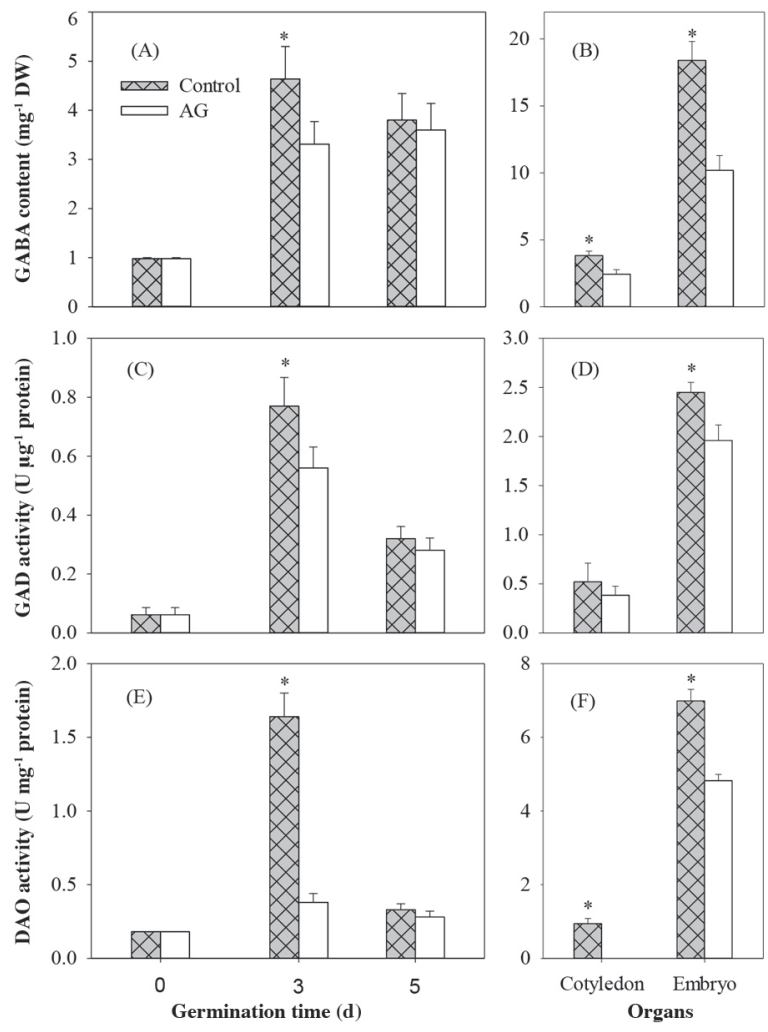

*Significant at $p<0.05$.

Figure 7. Effect of aminoguanidine (AG) on $\gamma$-aminobutyric acid (GABA) content (A, B), glutamate decarboxylase (GAD; C, D), and diamine oxidase (DAO; E, F) activity of germinating faba bean under $\mathrm{NaCl}$-hypoxia stress. Seeds germinating without $\mathrm{AG}$ were defined as the control. Indexes in the cotyledon and embryo of 3-d-old sprouts were determined. Each point was expressed as mean \pm SD.
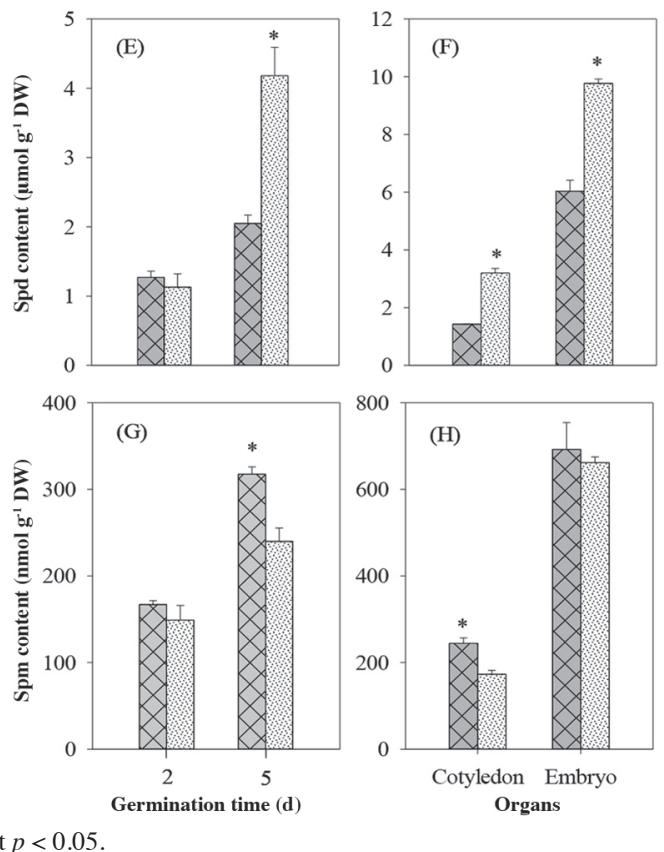

Figure 6. Effect of removing $\mathrm{NaCl}$ on glutamate (Glu; A, B) and polyamine $(\mathrm{C}, \mathrm{D}, \mathrm{E}, \mathrm{F}$, G, and $\mathrm{H})$ content of germinating faba bean under hypoxia. Seeds germinating under hypoxia were defined as the control. Indexes in the cotyledon and embryo were determined. Each point was expressed as mean \pm SD. 
the cotyledon as compared with the control (Figure $7 \mathrm{~F}$ ). However, no changes were observed in faba bean germinated for $5 \mathrm{~d}$ (Figure 7E).

When treated for $3 \mathrm{~d}$ with $\mathrm{AG}$, Glu content decreased significantly $(p<0.05)$ (Figure 8A), and mainly occurred in the embryo (Figure 8B). This was probably because the conversion of Glu to GABA and Pro was accelerated to meet the needs of resisting adversity when polyamine degradation pathway was inhibited. The Put content increased slightly under the AG treatment for $3 \mathrm{~d}$ (Figure $8 C$ ), while it increased in the cotyledon and decreased in the embryo (Figure 8D). Spermidine and Spm accumulated significantly with the prolongation of germination time (Figure 8E, 8G), as well as in both the cotyledon and embryo (Figure $8 \mathrm{~F}, 8 \mathrm{H}$ ) when treated for $3 \mathrm{~d}$.

\section{DISCUSSION}

\section{Effect of $\mathrm{NaCl}$ on GABA accumulation under hypoxia stress}

When compared with hypoxia (control), the $\mathrm{NaCl}$ treatment for $3 \mathrm{~d}$ promoted GABA and DAO activity. It was therefore verified that salt stress can improve GABA accumulation in the plant (Dittami et al., 2011). In contrast, GAD activity in the embryo decreased significantly under $\mathrm{NaCl}$ stress, indicating that DAO played a more important role for GABA accumulation in germinating faba bean. When treated with $\mathrm{NaCl}$ for $5 \mathrm{~d}$, GABA content and GAD and DAO activity decreased significantly. This was attributed to the enhanced intensity of stress by adding $\mathrm{NaCl}$, which strongly inhibited the physiological
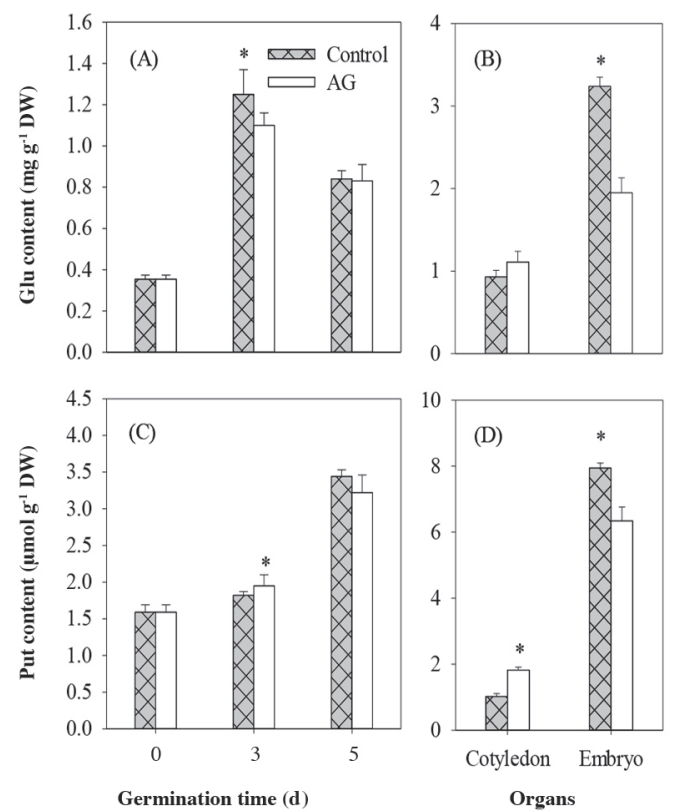

activity of germinating faba bean (Renault et al., 2010). When treated for a short time $(3 \mathrm{~d}), \mathrm{NaCl}$ induced the formation of soluble molecules (GABA, etc.) for resisting stress conditions (Xing et al., 2007; Renault et al., 2010). However, a longer stress time ( $5 \mathrm{~d}$ ) had a negative effect on seed survival and decreased enzyme activity, which retarded GABA accumulation. It was also found that GAD activity decreased significantly when treated with $\mathrm{NaCl}$ for $3 \mathrm{~d}$, and DAO activity decreased significantly when treated for $5 \mathrm{~d}$. This discrepancy indicated that the tolerance ability of GABA shunt to $\mathrm{NaCl}$ stress was lower than polyamine degradation pathway.

The plant needed more soluble molecules to fight against stressful conditions because of the increased stress intensity caused by adding $\mathrm{NaCl}$ under hypoxia. Glutamate was therefore converted into other soluble molecules to sustain physiological activity under $\mathrm{NaCl}$ stress for $3 \mathrm{~d}$. Polyamines (Put, Spd, and Spm) did not change significantly under $\mathrm{NaCl}$ stress for $3 \mathrm{~d}$, while they accumulated under strong stress for $5 \mathrm{~d}$ (Xing et al., 2007). This agreed with previous research that $\mathrm{NaCl}$ stress accelerated polyamine content (Anjum, 2010). Though polyamines are very important for GABA accumulation by polyamine degradation pathway, long stress time resulted in the suppression of physiological activity. Polyamine oxidase activity, including DAO, is therefore inhibited and polyamines are accumulated.

\section{Recovery from $\mathrm{NaCl}$ stress on GABA accumulation}

It was found that a long stress time treatment $(>3 \mathrm{~d})$ under hypoxia is more helpful for GABA accumulation
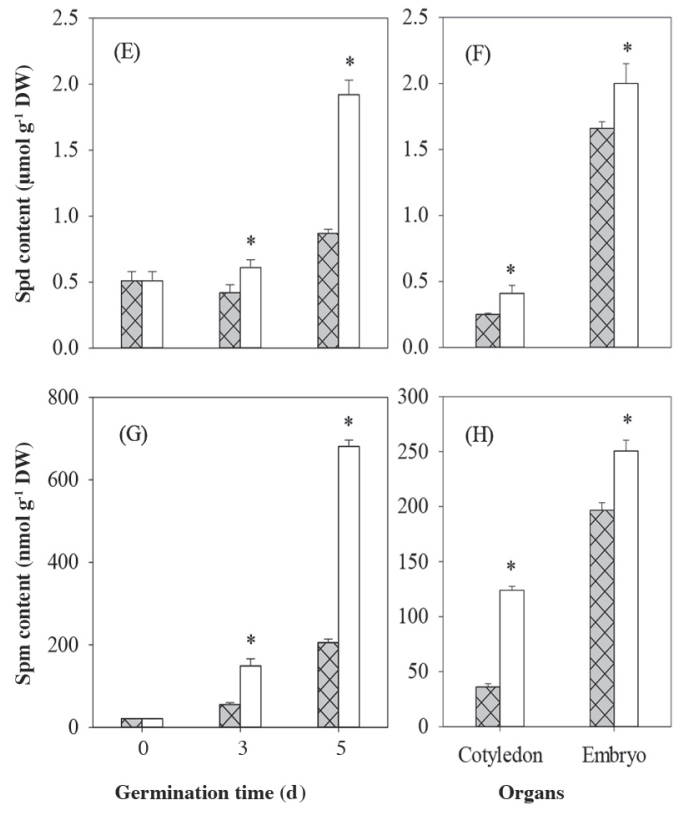

*Significant at $p<0.05$.

Figure 8. Effect of aminoguanidine (AG) on glutamate (Glu; A, B) and polyamine (C, D, E, F, G, and H) contents of germinating faba bean under hypoxia-NaCl stress. Seeds germinating without AG were defined as the control. Indexes in the cotyledon and embryo of 3-d-old sprouts were determined. Each point was expressed as mean \pm SD. 
than hypoxia- $\mathrm{NaCl}$ (Figure 3A). After recovering from $\mathrm{NaCl}$ stress, GABA content increased significantly in the embryo because physiological activity is vigorous and sensitive to recovery. Reducing stress by removing $\mathrm{NaCl}$ obviously decreased the high GABA level, essential for resisting adverse situations (Liu et al., 2011), to a normal level. Faba bean cotyledon is rich in nutrients that provide the substance and energy needed for embryo growth. Physiological activity in the cotyledon is weaker than in the embryo. After being treated with $\mathrm{NaCl}$ under hypoxia, cotyledon cell activity was further inhibited and even stagnant. Cotyledon stress was reduced when $\mathrm{NaCl}$ was removed, resulting in increased enzyme activity.

The Glu content had no significant changes after recovery. The reason might be that the removal of $\mathrm{NaCl}$ enhanced sprout physiological activity, so that protease activity increased (Kirmizi and Güleryüz, 2006) and thus degraded proteins to produce Glu. Simultaneously, Glu was degraded into nitrogen, carbon skeleton, and energy to support the formation and development of new organs. Polyamines are produced from amino acids (especially arginine) (Gomez-Jimenez et al., 2010) and converted by polyamine oxidases (including DAO) into GABA via a $\gamma$-aminobutyl aldehyde intermediate. Xing et al. (2007) also found that polyamine content in soybean root increased significantly after recovery from $\mathrm{NaCl}$ stress. It might be related to the enhancement of physiological activity after recovery.

\section{Effect of AG on GABA accumulation under hypoxia- NaCl stress}

Adding AG under hypoxia- $\mathrm{NaCl}$ stress reduced GABA production and mainly occurred in the embryo. The contribution ratio of polyamine degradation pathway for GABA accumulation was $29.82 \%$, at least when treated for $3 \mathrm{~d}$ under hypoxia- $\mathrm{NaCl}$, because $\mathrm{DAO}$ activity was not completely inhibited (Figure 7C). Hypoxia$\mathrm{NaCl}$ treatment for a short time $(3 \mathrm{~d})$ could raise the contribution ratio of polyamine degradation pathway on GABA accumulation in germinating faba bean. The long stress time ( $>3 \mathrm{~d}$ ) treatment neither helped GABA accumulation nor enhanced the contribution ratio of polyamine degradation pathway for GABA accumulation. The faba bean embryo was sensitive to adding AG; GABA content and enzyme activity decreased significantly.

When polyamine degradation was blocked by AG, Glu degradation significantly increased GABA and Pro content against the stressful condition. Hence, adding AG resulted in decreasing Glu content in 3-d-old sprouts. In 5-d-old sprouts, there was no significant difference in Glu content between the AG treated group and the control. However, it decreased significantly when compared with 3-d-old treated sprouts. Sprout physiological activity decreased after being treated by hypoxia- $\mathrm{NaCl}$ for a long time (5 d), which slowed down or even stopped Glu production. Based on the above, we found that GAD tolerance to high intensity stress (hypoxia- $\mathrm{NaCl}$ ) was weaker. Compared with GAD, DAO was more tolerant to $\mathrm{NaCl}$ stress. Polyamine oxidation could therefore be performed efficiently under hypoxia- $\mathrm{NaCl}$ stress. The DAO activity was inhibited strongly when AG was added, which prevented polyamine degradation and resulted in Put accumulation. The DAO in faba bean could degrade Spd a little, but could only degrade Spm when it was converted into Put (Yang et al., 2012). Hence, Spd and Spm were accumulated as a result of inhibiting DAO activity.

\section{CONCLUSIONS}

Hypoxia treatment should be used to accelerate GABA accumulation by DAO synthetic pathway during a short time action of salt stress. The damaging effect of polyamine accumulation during salt stress is thus avoided.

\section{ACKNOWLEDGEMENTS}

We are grateful for the financial support of the Natural Science Foundation of China (31401614) and a project funded by the Priority Academic Program Development of Jiangsu Higher Education Institutions (PAPD).

\section{LITERATURE CITED}

Anjum, M.A. 2010. Response of Cleopatra mandarin seedlings to a polyamine-biosynthesis inhibitor under salt stress. Acta Physiologiae Plantarum 32:951-959.

Azeke, M.A., R.M. Elsanhoty, S.J. Egielewa, and M.U. Eigbogbo. 2011. The effect of germination on the phytase activity, phytate and total phosphorus contents of some Nigerian-grown grain legumes. Journal of the Science of Food and Agriculture 91:7579.

Bai, Q.Y., M.Q. Chai, Z.X. Gu, X.H. Cao, Y. Li, and K.L. Liu. 2009. Effects of components in culture medium on glutamate decarboxylase activity and gamma-aminobutyric acid accumulation in foxtail millet (Setaria italica L.) during germination. Food Chemistry 116:152-157.

Bhatnagar, P., B.M. Glasheen, S.K. Bains, S.L. Long, R. Minocha, C. Walter, et al. 2001. Transgenic manipulation of the metabolism of polyamines in poplar cells. Plant Physiology 125:2139-2153.

Bouche, N., B. Lacombe, and H. Fromm. 2003. GABA signaling: a conserved and ubiquitous mechanism. Trends in Cell Biology 13:607-610.

Bown, A.W., and B.J. Shelp. 1997. The metabolism and functions of $\gamma$-aminobutyric acid. Plant Physiology 115:1-5.

Dittami, S.M., A. Gravot, D. Renault, S. Goulitquer, A. Eggert, A Bouchereau, et al. 2011. Integrative analysis of metabolite and transcript abundance during the short-term response to saline and oxidative stress in the brown alga Ectocarpus siliculosus. Plant Cell and Environment 34:629-642.

Gomez-Jimenez, M.C., M.A. Paredes, M. Gallardo, and I.M. Sanchez-Calle. 2010. Mature fruit abscission is associated with up-regulation of polyamine metabolism in the olive abscission zone. Journal of Plant Physiology 167:1432-1441.

Goyoaga, C., C. Burbano, C. Cuadrado, A. Varela, E. Guillamón, M.M. Pedrosa, et al. 2008. Content and distribution of vicine, convicine and L-DOPA during germination and seedling growth of two Vicia faba L. varieties. European Food Research and Technology 227:1537-1542. 
Guillamon, E., M.M. Pedrosa, C. Burbano, C. Cuadrado, M.D.C. Sanchez, and M. Muzquiz. 2008. The trypsin inhibitors present in seed of different grain legume species and cultivar. Food Chemistry 107:68-74.

Guo, Y., H. Chen, Y. Song, and Z. Gu. 2011. Effects of soaking and aeration treatment on $\gamma$-aminobutyric acid accumulation in germinated soybean (Glycine max L.) European Food Research and Technology 232:787-795.

Guo, Y., R. Yang, H. Chen, Y. Song, and Z. Gu. 2012. Accumulation of $\gamma$-aminobutyric acid in germinated soybean (Glycine max L.) in relation to glutamate decarboxylase and diamine oxidase activity induced by additives under hypoxia. European Food Research and Technology 234:679-687.

Kinnersley, A.M., and F.J. Turano. 2000. Gamma aminobutyric acid (GABA) and plant responses to stress. Critical Reviews in Plant Sciences 19:479-509.

Kirmizi, S., and G. Güleryüz. 2006. Protein mobilization and proteolytic enzyme activities during seed germination of broad bean (Vicia faba L.) Zeitschrift Fur Naturforschung C-A Journal of Biosciences 61:222-226.

Li, Y., Q. Bai, X. Jin, H. Wen, and Z. Gu. 2010. Effects of cultivar and culture conditions on $\gamma$-aminobutyric acid accumulation in germinated fava beans (Vicia faba L.) Journal of the Science of Food and Agriculture 90:52-57.

Liu, C., L. Zhao, and G. Yu. 2011. The dominant glutamic acid metabolic flux to produce g-amino butyric acid over proline in Nicotiana tabacum leaves under water stress relates to its significant role of antioxidant activity. Journal of Integrative Plant Biology 53:608-618.

Misra, A.N., A.K. Biswal, and M. Misra. 2002. Physiological, biochemical and molecular aspects of water stress responses in plants, and the biotechnological applications. Proceedings of the National Academy of Sciences India Section B 72 (2):115-134.

Mody, I., Y. De Koninck, T. Otis, and I. Soltesz. 1994. Bridging the cleft at GABA synapses in the brain. Trends in Neurosciences $17: 517-525$
Oomah, B.D., G. Luc, C. Leprelle, J.C.G. Drover, J.E. Harrison, and M. Olson. 2011. Phenolics, phytic acid, and phytase in Canadiangrown low-tannin faba bean (Vicia faba L.) genotypes. Journal of Agricultural and Food Chemistry 59:3763-3771.

Renault, H., V. Roussel, A. El Amrani, M. Arzel, D. Renault, A. Bouchereau, et al. 2010. The Arabidopsis pop2-1 mutant reveals the involvement of GABA transaminase in salt stress tolerance. BMC Plant Biology 10:20.

Vilariño, M., J.P. Métayer, K. Crépon, and G. Duc. 2009. Effects of varying vicine, convicine and tannin contents of faba bean seeds (Vicia faba L.) on nutritional values for broiler chicken. Animal Feed Science and Technology 150:114-121.

Wellner, A., C. Huettl, and T. Henle. 2011. Formation of Maillard reaction products during heat treatment of carrots. Journal of Agricultural and Food Chemistry 59:7992-7998.

Xing, S.G., Y.B. Jun, Z.W. Hau, and L.Y. Liang. 2007. Higher accumulation of gamma-aminobutyric acid induced by salt stress through stimulating the activity of diamine oxidases in Glycine max (L.) Merr. roots. Plant Physiology and Biochemistry 45:560-566.

Yang, R., H. Chen, and Z. Gu. 2011a. Factors influencing diamine oxidase activity and $\gamma$-aminobutyric acid content of fava bean (Vicia faba L.) during germination. Journal of Agricultural and Food Chemistry 59:11616-11620.

Yang, R., H. Chen, Y. Han, and Z. Gu. 2012. Purification of diamine oxidase and its properties in germinated fava bean (Vicia faba L.) Journal of the Science of Food and Agriculture 92:1709-1715.

Yang, R., Q. Guo, and Z. Gu. 2013. GABA shunt and polyamine degradation pathway on $\gamma$-aminobutyric acid accumulation in germinating fava bean (Vicia faba L.) under hypoxia. Food Chemistry 136:152-159.

Yang, R., J. Song, Z. Gu, and C. Li. 2011b. Partial purification and characterisation of cysteine protease in wheat germ. Journal of the Science of Food and Agriculture 91:2437-2442. 